\title{
IDENTIFICATION OF RESILIENCE FACTORS IN RAIL ENGINEERING WORK
}

\author{
${ }^{12}$ Pedro Ferreira Pedro.Ferreira@networkrail.co.uk \\ ${ }^{1}$ Theresa Clarke Theresa.Clarke@networkrail.co.uk \\ ${ }^{12}$ John Wilson John.Wilson@nottingham.ac.uk \\ ${ }^{2}$ Sarah Sharples Sarah.Sharples@nottingham.ac.uk \\ ${ }^{2}$ Brendan Ryan Brendan.Ryan@nottingham.ac.uk \\ ${ }^{1}$ Network Rail - 40, Melton Street - London NW1 2EE UK \\ ${ }^{2}$ Human Factors Research Group - Faculty of Engineering - University of \\ Nottingham, University Park, Nottingham NG7 2RD, UK
}

\begin{abstract}
This paper discusses an ongoing project that aims at improving the potential for resilience of a system responsible for the planning of rail engineering work delivery. It focuses on the use of a human factors based approach as a way to achieve this end. In particular, the paper discusses the initial data collected by means of interviews and how this process gave way to a two fold goal: Understanding how the planning process works in reality and identifying any critical aspects of the system from a Resilience Engineering perspective.

Given the nature of the process under study, information flows and communication issues have been given particular attention throughout the data collection and analysis stages. Initial data confirms that the planning process is greatly reliant on the capability of people using their knowledge and skills to communicate in a dynamic informational environment.

Finally, the added value of the interviews is discussed from a human factors perspective and as a mean towards the aim of better understanding resilience in rail engineering planning.
\end{abstract}

\section{Introduction}

Network Rail as the owner of rail infrastructure in the UK, is currently being submitted to a considerable pressure from governance and the wither public to deliver capacity increments to the network on a scale never experienced before. Within this context, the planning of engineering work operates under high variability and complexity conditions and faces a considerable exposure to business and operational pressures as it deals directly with critical decisions regarding the delivery of Network Rail's service to its customers (train operators). Earlier work (Ferreira et al 2008) has discussed these issues and demonstrated the potential interest of Resilience Engineering (Wreathall 2006) approaches to tackle these challenges.

Wreathall (2006) defines resilience as the ability of a system to keep, or recover quickly, to a stable state, allowing it to continue operations during and after a major mishap or in the presence of continuous significant stresses. As extensively discussed in Hollnagel et al (2006), this ability of the system resides a great deal in pursuing an adequate balance between the pressures for higher productivity and the assurance of the required safety standards.

The planning of rail engineering work, in a simplistic way, can be described as a system that aims to schedule the delivery of all work requests within a calendar year, whilst optimising the integration and usage of available resources, of which access to the infrastructure could be considered the most critical one. Although planning begins about 90 weeks before foreseen date of delivery, many work requests are brought in at various stages of the process in a response to unforeseeable infrastructural and business demands. Each work item planned is then delivered within a worksite, which is integrated and protected under arrangements designated as possessions 
(taking control of a section of track for engineering works). A significant volume of less complex maintenance work (in general, those that do not affect the safe running of trains) is often delivered under less complex protection arrangements.

The ongoing research project discussed in Ferreira et al (2008) proposes Resilience Engineering as a theoretical background to develop a better understanding of how the increasing complexity of planning may be efficiently and safely managed. This paper brings further developments towards this end and explores more in depth, two different initial questions:

1. How does the planning process work in reality beyond its formal description?

2. What are the critical aspects of the system from a resilience perspective?

\section{Method}

Given the high complexity and broadness of the field under study, several approaches to data collection and analysis are being used, aiming to develop a better understanding of the real planning activities and their interactions. Five different analysis steps are being undertaken throughout this project:

1. Interviews

2. Analysis of safety data

3. Analysis of historic planning data

4. Observation of planning meetings

5. Observation of engineering work delivery (track work)

The information provided in this paper, although mostly based on the outcome of the interview process, draws on preliminary data collected in the course of the other on going stages.

The interviews were based on a set of ten questions (table 1) and recorded for later transcription and analysis. The analysis focused more on concepts and ideas given by interviewees, to identify major trends and give direction for more in-depth analyses. To avoid any distortion of information, transcriptions were reviewed and checked against original recordings. Whenever necessary sentences were transcribed and in some cases, the person interviewed was contacted again for additional clarification.

Table 1: Topics that formed the basis of the interviews

Can you tell me about your job (daily tasks, duties)?

Can you explain the planning process from your point of view? How would you describe your influence/role in the process?

How often would you say your job changes (How and what changes)?

Do you normally feel confident regarding the outcome of the planning activities in which you take part? Can you identify any recurrent uncertainties that you are faced with?

What would you say are your main skills and competencies?

How would you describe the overall set of rules and procedures applicable to the planning process?

What type of information do you use most? How important is it in your job?

What do you consider to be the current major challenges for track work delivery? How does the planning process respond to those challenges?

What does resilience mean to you?

Throughout your professional experience in this area, what success stories come to your mind? What failures? 


\section{Results}

The duration of interviews varied from about 50 minutes up to nearly two hours, depending on how interviewees elaborated on each subject. About 16 hours of recordings were made for a total of 13 interviews, of which 7 were held with managers of planning units at area and territory levels (geographical structures for Network Rail).

The age of interviewees ranged from late 20 s to $50 \mathrm{~s}$, with an average placed between 30 and 35 years old. Even the younger planners had at least three years of experience in planning and the majority had 10 or more years of experience in different roles and levels of the rail engineering industry.

The information provided by the interview process is thoroughly explored in Ferreira et al (2008). The issues presently under discussion focus on factors relevant for the potential for resilience of the system. Table 2 categorises these issues based on whether they contribute for the enhancement or the erosion of the potential for resilience. Each of these issues is then further clarified and substantiated by data extracted from the interview process.

Table 2: Factors that influence the potential for resilience

\begin{tabular}{|c|c|}
\hline Erosion of the potential for resilience & $\begin{array}{l}\text { Enhancement of the potential for } \\
\text { resilience }\end{array}$ \\
\hline Organisational breakdowns & Planning experience and skills \\
\hline Variability of inputs from stakeholders & Solid work relations \\
\hline Poor information flows & Informal communication \\
\hline High uncertainty and unpredictability & Understanding the impact of decisions \\
\hline \multicolumn{2}{|c|}{ Planning decisions (trade-offs) } \\
\hline Frequent planning changes & Development of contingency solutions \\
\hline
\end{tabular}

\subsection{Planning decisions}

Throughout the interviews, planners frequently stressed the importance of communication and access to reliable information as a fundamental support for decision making. The planning process is essentially a system that produces information with the purpose of supporting engineering work delivery. This confers a crucial role to the information flows within the system, as these are the basis of every decision made by planners throughout the process. These decisions in effect, are the means through which the system balances the work requests against the available track access.

From a Resilience Engineering perspective, the decision processes ${ }^{1}$ of planners are the element that shapes the trade-offs of the system. Within this frame, planning decisions were considered the element with the most direct influence over the potential for resilience, as they determine how adequately the system is able to balance productivity pressures against safety requirements (Hollnagel et al 2006). The more planners are able to steer their decision process towards optimising access opportunities without compromising the safety and reliability of work delivery, the higher the potential for resilience within the planning system. Also within this perspective, the issues referred to in table 2 can be considered as factors that either support or hamper the decision processes of planners.

\footnotetext{
${ }^{1}$ The decision process refers to the "external" means and strategies utilised by planners to reach a decision. It is used instead of "decision making" as this usually refers to the more "internal" cognitive aspects of reaching a decision.
} 


\subsection{Organisational breakdowns}

The organisational structure that supports the planning process is divided into several levels and functions, which creates difficulties in the exchange of information and coordination of needs and goals. The breakdown of the process, attributed by planners to the fact that it operates divided between investments (Corporate function responsible for most of the infrastructure renewals and enhancements) and maintenance (Corporate function responsible for maintaining the infrastructure) as well as territory and area level teams (figure 1), was mentioned as a major obstruction to planning efficiency and reliability.

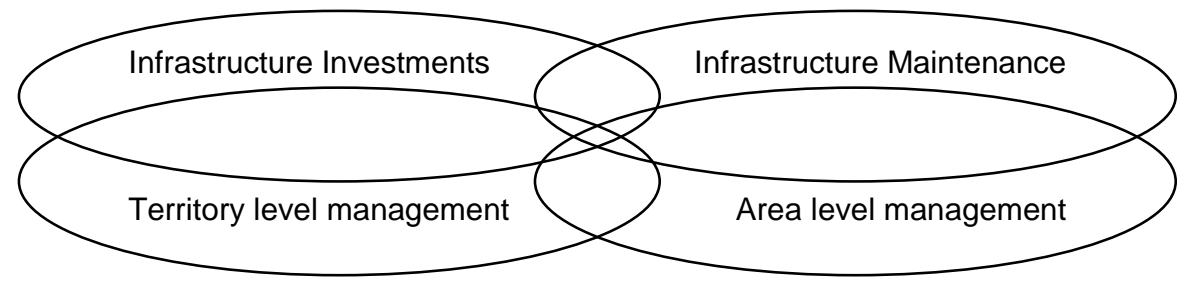

Fig. 1: Main organisational functions and levels of management that compose the planning system. Within each of these structures, independent teams develop specific stages of the planning process, which then exchange information at formally pre-defined timescales.

The work scopes and priorities of these organisational structures don't necessarily coincide. Because of insufficient track access opportunities to respond to all work demands, investments and maintenance often compete with each other for priority. Planners admitted that the fragmentation of the process intensifies this competition, leading to occasional conflicts and tensions, as each team attempts to minimise the impacts of business and operation pressures over their own performance.

The planning system not only operates in the connection of several functions and levels within Network Rail, but it also relies on other organisations to provide crucial information as an input to planning decisions.

\subsection{Variability of inputs from stakeholders}

Interviewed planners stated that stakeholders inputting to planning (Investments maintenance, contractors, among others) tend to use different contents and formats for the information they provide to planning, particularly at the initial stages of the planning process when only general work scopes are being submitted. Beyond this point and throughout the entire process, planners also have to deal with differences in time lines of each stakeholder, as progressively more detailed and complex plans are being developed. Planners referred to this high input variability as a major cause of time loss cross-checking information and of some frustration due to the duplication of work in which it often incurs. This also creates a potential for planning failure as variability in communication can lead to inaccuracies and omissions of planning details.

\subsection{Poor information flows}

The planning organisation relies on inputs from all areas and functions of network Rail and its contractors. A complex structure of input and feedback information is necessary to achieve optimised planning decisions and make the most of available resources locally as well as nationally. This generates interdependency between all functions and levels of planning, which can act against the need for prompt and accurate information to support decision processes. This is mainly substantiated by the poor visibility and information that planners claimed regarding how the system performs outside their working teams.

Planners often referred to the communication with stakeholders outside Network Rail as being too complex and time consuming. Given the significant input to the 
planning process of organisations such as engineering contractors, this constitutes a clear barrier to the efficiency and reliability of planning decisions.

\subsection{Uncertainty and unpredictability}

High organisational complexity hampers planners' ability to anticipate the system's behaviours. Dealing with uncertainty and unpredictability was recognised by all interviewees as part of the daily activity in planning.

Planning changes were mentioned by all planners interviewed as the main cause of variability and unpredictability of the job. These changes emerge as the need to review planning decisions already made. Although not necessarily affecting their ability to develop a robust plan, this was also considered the main deterrent factor of confidence in the performance of the whole planning organisation. The majority of planners tended to consider that changes are more or less directly originated by priorities that emanate from company board level. These create budget and work scope changes for work items already undergoing planning and cause knock-on effects throughout the entire planning structure. Because changes normally emanate from outside the planning structure, it is beyond the control of planners to try and manage these effectively and minimise impacts over the robustness of the plan.

\subsection{Frequent planning changes}

Difficulties in coordinating a high diversity of engineering work demands within limited access resources generate high pressures on planning to maximise every access opportunity. This is achieved through complex negotiations between all work deliverers as a way to find the best possible compromise for all.

The issue under discussion here concerns not planning changes themselves but rather the frequency with which they tend to occur. In fact, changes can either contribute to the robustness and coherence of planning or the deterioration of it. When a given planned job is submitted to frequent and numerous changes, it is certain that not only its coherence and integrity are threatened but also maintaining and communicating accurate and up-to-date information regarding those changes becomes increasingly difficult.

\subsection{Planning experience and skills}

The absence of any specific training for planning and the complexity and scale of the system makes experience and personal skills a valuable resource to achieve successful planning solutions.

Experience and overall industry knowledge were mentioned by all planners as an important resource to realise what information is required at each step to support planning decisions. According to planners this enables an understanding of what the impact of those decisions might be on the day of delivery. Experience also becomes relevant for the building of solid relationships with stakeholders. By knowing how people work and respond under different circumstances, planners are able to anticipate potential problems.

Understanding how the rail industry works as whole together with experience enables planners to better balance their decisions, either by introducing safeguards, restrictions or degrees of freedom to the plan, according to their perception of each situation and work request.

\subsection{Solid work relations}

Trusting people in key planning roles supports decision processes by facilitating access to reliable information. These relationships establish levels of confidence and mutual trust that support informal discussion of issues and problem solving.

Despite any occasional diverging needs, all planners recognised cooperation and teamwork as a fundamental basis for the development of a robust and nationally integrated delivery plan. Solid work relations provide grounds for an informal but fast 
information sharing structure that is fundamental to overcome planning conflicts without having to wait for any formal timescales and documentation to reach a solution. This becomes even more important when finding those solutions requires reaching an agreement with parties outside Network Rail such as contractors or train operators.

\subsection{Informal communication}

Knowing who to contact to obtain reliable information or confirmation on other sources represents a significant contribute to successful planning.

All interviewees pointed out information and communication channels as a crucial resource for planning. One planner stated that the job consisted on collecting and delivering information for $90 \%-95 \%$ of the day. In particular, several planners stated that efficient communication of any late changes occurring at pre-possession stage (usually three days before delivery) is crucial to prevent a possession from overrunning or even causing serious losses or injuries.

All interviewees mentioned the frequent need to cross check and chase accurate information. This is accomplished through informal contacts with people within stakeholders they feel they can rely on. All planners valued the work relations that support this informal communication and tend to go to them, rather than relying on the organisation and its formal communication channels.

\subsection{Understanding the impact of planning decisions}

Knowing how much work and what type of work it is safe to integrate into each access opportunity requires understanding how planning decisions may affect work delivery.

Planners have demonstrated being aware of the potential impacts of their decisions over work delivery, regardless of the difficulties created by poor visibility of the process as a whole. This awareness is developed through both experience and the informal communication held with all those inputting to planning as a way to confirm their understanding of each delivery scenario. Only with a sufficiently clear understanding of work delivery can planners have foresight of potential problems and integrate safeguards or work limitations they see fit to achieve a robust plan.

\subsection{Contingency planning}

Supporting work delivery in being prepared for any delays or equipment failures that may arise, particularly in complex possessions contributes to robustness of planning.

Job experience and overall understanding of the railways were considered by the majority of planners as decisive, not only to explore different possible contingencies (i.e. different ways of delivering work) but also to adequately integrate these within the planning process (i.e. anticipate and solve potential conflicts). Planners know that some contractors tend to fail more than others in terms of reliability and quality of work. When the possession manager (organisation or function that owns the work requested) is someone they know to be less reliable, planners try to allow additional spare time at the end of the possession for problem recovery (contingency plan).

\section{Discussion}

As previously mentioned, Ferreira et al (2008) has addressed the way business pressures impact on the planning system. Planners generally expressed this pressure through the need to solve conflicts created by insufficient access to respond to all requests for maintenance, renewals and enhancement works. The data so far gathered in the course of this project stresses the crucial role of planners' decisions in balancing these pressures against safety requirements. As Dekker (2006) states, Resilience Engineering can provide useful contribution towards understanding how these "micro-level" decisions are linked to the "macro-level" drift into failure of 
systems. In other words, making progress on the potential for resilience requires understanding how the pressures at macro-level affect the decision processes at micro-level and in return, how these micro-level decisions impact the systems "behaviours" as whole. The evidence of strong relations between all the previously discussed issues provides useful insight towards understanding this.

Amongst the factors that erode the potential for resilience, as well as amongst those that contribute to its improvement, evidence of direct interactions was found. Not only the information flows have shown to be crucial to this system, but also their poor quality appears to generate critical interactions between the eroding factors. This means that for example, the more variable become the inputs from stakeholders, the poorer the information flows tend to be and the higher the unpredictability. In return, this higher unpredictability will tend to deteriorate the information flows that work as a feedback from planning to stakeholders and thus contributing to further degradation of their input. Similar examples can be given amongst the factors contributing to improved resilience. Again focusing on the importance of information, the informal communication was shown to be supported by solid work relations. In return, these work relations are built on mutual trust that emerges from the exchange of information and the support that planners tend to provide to each other. Thus, if the informal communication between planning teams was to deteriorate the working relations would tend to become less reliable and it also likely that this would negatively impact on the ability of planners to learn from experience and hence, to foresee the consequences of their decisions.

This data also substantiates the existence of interactions between factors eroding on the potential for resilience and those contributing to its improvement. Planners attempt to balance each of the factors that hinder resilience by making use of a specific resource. For instance, planners make use of their experience and overall knowledge of the industry to deal with the constraints imposed by organisational breakdowns. In the same way, Informal communication is directly aimed at minimising the impacts of the poor information formally provided by the process. Thus, each of the factors contributing to the enhancement of resilience corresponds to a specific resource planners mentioned as being fundamental to achieve reliable and safe planning. Table 2 shows each of these resources opposite to the resilience hindering factor to which planners pointed out as trying to compensate for.

\section{Conclusions}

The progress of this on going study has continuously brought in depth knowledge, not only regarding what constrains and what facilitates the reliability of planning, but also how Resilience Engineering concepts may contribute to better manage these factors. As mentioned by Ferreira et al (2008), although the consequences of planning failure are rarely associated with the risk of human losses, there's a considerable risk for loss of work opportunities and resources which can result in serious financial loss and the compromise of the rail infrastructure's integrity. Within this context, research based on resilience engineering concepts seems relevant.

The interviewing process gave way to an in depth knowledge of the planning components and their interactions. Understanding what affects planners' decisions means knowing how people work in reality and how they deal with the constraint imposed by the external pressures created at system level. It has then proved to be a valuable tool towards the purpose of understanding how the interactions between micro and macro levels take place within the planning system.

\section{References}

Amalberti, R. (2001). The paradoxes of almost totally safe transportation systems. Safety Science, 37, pp. 109-126 
Dekker, S. (2006). Resilience Engineering: Chronicling the emergence of confused consensus. In Hollnagel, E., Woods, D.D., Leveson, N. Resilience Engineering - Concepts and Precepts. (pp 77 - 92) Aldershot, UK: Ashgate

Ferreira, P., Clarke, T., Wilson, J., Sharples, S., Ryan, B., (2008). Resilience in rail engineering work. In Hollnagel, E., Pieri, F., Rigaud, E., Proceedings of the third Resilience Engineering Symposium. Ecole des mines de Paris, Paris: France

Hollnagel, E. (2004). Barriers and accident prevention. Aldershot, UK: Ashgate

Hollnagel, E., Woods, D.D., Leveson, N. (2006) Resilience Engineering Concepts and Precepts. Aldershot, UK: Ashgate

Woods, D.D., Hollnagel, E. (2006). Prologue: Resilience Engineering Concepts. In Hollnagel, E., Woods, D.D., Leveson, N. Resilience Engineering - Concepts and Precepts. (pp 1 - 6) Aldershot, UK: Ashgate

Wreathall, J. (2006). Properties of resilient organizations: An initial view. In Hollnagel, E., Woods, D.D., Leveson, N. Resilience Engineering - Concepts and Precepts. (pp 275 - 285) Aldershot, UK: Ashgate 